PROCEEDINGS OF THE

AMERICAN MATHEMATICAL SOCIETY

Volume 131, Number 8, Pages 2347-2358

S 0002-9939(03)07077-1

Article electronically published on March 18, 2003

\title{
THE STRONG OPEN SET CONDITION FOR SELF-CONFORMAL RANDOM FRACTALS
}

\author{
NORBERT PATZSCHKE
}

(Communicated by David Preiss)

\begin{abstract}
We prove that the open set condition and the strong open set condition are equivalent for self-conformal random fractals.
\end{abstract}

\section{INTRODUCTION}

In the theory of fractal sets and measures, self-similar sets have been the most studied. A set $X$ is called self-similar if it is invariant under a family of similarities $S_{1}, \ldots, S_{N}$, that is, if $X=\bigcup_{i=1}^{N} S_{i}(X)$. If one allows the mappings $S_{i}$ to be conformal ones, $X$ is called a self-conformal set. If the family $S_{1}, \ldots, S_{N}$ satisfies the open set condition, that is, if there is an open set $O$ such that $S_{i}(O) \subseteq O$ for all $i$ and $S_{i}(O) \cap S_{j}(O)=\emptyset$ for $i \neq j$, one can compute some characteristics of it. For instance, in the case of similarities the Hausdorff dimension of the self-similar set equals the similarity dimension.

If one considers measures instead of sets, for example a self-similar or selfconformal measure $\mu$ with the property $\mu=\sum_{i=1}^{N} p_{i} \cdot \mu \circ S_{i}^{-1}$, it is useful that the strong open set condition is satisfied, that is, in addition to the open set condition $X \cap O \neq \emptyset$ holds. For the case when all mappings are similarities, Schief [S] proved that both conditions are equivalent. For self-conformal sets the problem was open for a long time. Recently, Peres, Rams, Simon, and Solomyak [PRSS] and Lau, Rao, and Ye [LRY] proved the equivalence in the self-conformal case.

By allowing the mappings $S_{i}$ to be random mappings, one can define random fractals. A random compact set $\Xi$ is a self-conformal random set if it has the same distribution as $\bigcup_{i=1}^{N} S_{i}\left(\Xi^{i}\right)$, where the $\Xi^{i}$ are independent copies of $\Xi$ and are independent of $\left(S_{1}, \ldots, S_{N}\right)$. In other words, a self-conformal random set $\Xi$ consists of $N$ parts, which are conformal copies of independent samples of $\Xi$. The self-conformal random sets are also known as statistically self-conformal sets.

The equivalence of the open set condition and the strong open set condition for self-similar random sets is proved in $[\mathrm{P} 1$ by varying the proof of Schief $\mathrm{S}$. In the present paper we will prove that it also holds for self-conformal random sets.

The paper is organized as follows. First, we give a definition of the term selfconformal random set and give a method for constructing such sets. The definition is more or less a combination of the known definitions for self-conformal sets and

Received by the editors March 6, 2001 and, in revised form, August 30, 2001.

2000 Mathematics Subject Classification. Primary 28A80; Secondary 60D05, 60G57.

Key words and phrases. Random fractals, (strong) open set condition.

(C)2003 American Mathematical Society 
self-similar random sets. For more details concerning some notations the reader is referred to $[\mathrm{P} 2]$ and $[\mathrm{AP}$.

The main part of this article is the proof of the equivalence of the open set condition and the strong open set condition. At first it is shown that there is a random open set which satisfies the strong open set condition. The proof follows the proof given by Peres, Rams, Simon, and Solomyak [PRSS, but some effort is necessary because of the randomness. Then, using this random open set, a nonrandom set is constructed such that the strong open set is fulfilled.

\section{SELF-CONFORMAL RANDOM FRACTALS}

Let $U \subseteq \mathbb{R}^{d}$ be an open bounded connected subset. For $0<\gamma \leq 1$ denote by $\operatorname{Con}^{1+\gamma}(U)$ the family of conformal diffeomorphisms $S: U \rightarrow S(U) \subseteq U$ for which there exists a constant $C_{S}$ such that

$$
\left|S^{\prime}(y)-S^{\prime}(x)\right| \leq C_{S} d(x, y)^{\gamma}
$$

holds for all $x, y \in U$, where $S^{\prime}(x)$ is the differential of $S$ at $x$ and $\left|S^{\prime}(x)\right|$ is the operator norm of this differential. Since $S$ is a conformal mapping, we have $\left|S^{\prime}(x) v\right|=\left|S^{\prime}(x) \| v\right|$ for all $v \in T_{x} U$.

Let $N \geq 2$ be a positive integer. Consider the product space

$$
\Omega_{0}=\left(\operatorname{Con}^{1+\gamma}(U)\right)^{N} .
$$

It is a separable metrisable space with the Borel $\sigma$-algebra $\mathfrak{F}_{0}$.

We say that a probability measure $\mathbb{P}_{0}$ on $\left(\Omega_{0}, \mathfrak{F}_{0}\right)$ is a conformal random function system if there are constants $0<r_{\min } \leq r_{\max }<1$ and a constant $C_{0}>0$ such that

(I) $C_{S_{i}} \leq C_{0}$ for all $i=1, \ldots, N$ and

(II) $r_{\min } \leq\left|S_{i}^{\prime}(x)\right| \leq r_{\max }$ for all $x \in U$ and all $i=1, \ldots, N$

hold for $\mathbb{P}_{0}$-almost all $\left(S_{1}, \ldots, S_{N}\right)$.

A random compact set $\Xi \subseteq U$ is called a self-conformal random set associated with the conformal random function system $\mathbb{P}_{0}$ if $\Xi$ has the same distribution as

$$
\bigcup_{i=1}^{N} S_{i} \Xi^{i}
$$

where the $\Xi^{i}, i=1, \ldots, N$, are independent samples of $\Xi$ and are independent of $\left(S_{1}, \ldots, S_{N}\right)$, and where $\left(S_{1}, \ldots, S_{N}\right)$ is distributed according to $\mathbb{P}_{0}$. That is, if $\mathbb{P}_{\Xi}$ is a distribution of a random compact set, then it is a distribution of a self-conformal random set, iff $\mathbb{P}_{\Xi}=\left(\mathbb{P}_{0} \times \mathbb{P}_{\Xi}^{N}\right) \circ u^{-1}$, where

$$
u\left(\left(S_{1}, \ldots, S_{N}\right) ; X^{1}, \ldots, X^{N}\right)=\bigcup_{i=1}^{N} S_{i}\left(X^{i}\right) .
$$

In the case when the $S_{i}$ are similarities we speak of self-similar random sets. It is well known (see $[\underline{F},[\underline{G},[\underline{M W}$ ) that, given a conformal random function system, there exists a unique distribution of a self-conformal random set $\Xi$.

The conformal random function system $\mathbb{P}_{0}$ is said to satisfy the open set condition (OSC) if there is an open set $O$ with $\bar{O} \subseteq U$ such that

(I) $S_{i}(O) \subseteq O$ for all $i=1, \ldots, N$ and

(II) $S_{i}(O) \cap S_{j}(O)=\emptyset$ for all $i \neq j$ hold for $\mathbb{P}_{0}$-almost all $\left(S_{1}, \ldots, S_{N}\right)$. 
$\mathbb{P}_{0}$ satisfies the strong open set condition (SOSC), if there is an open set $O$ with $\bar{O} \subseteq U$ and

(I) $S_{i}(O) \subseteq O$ for all $i=1, \ldots, N$ and

(II) $S_{i}(O) \cap S_{j}(O)=\emptyset$ for all $i \neq j$

for $\mathbb{P}_{0}$-almost all $\left(S_{1}, \ldots, S_{N}\right)$, and

(III) $\Xi \cap O \neq \emptyset$ with probability one,

where $\Xi$ is the self-conformal random set.

Since the random mappings $S_{i}$ are diffeomorphisms we may assume without loss of generality that $\operatorname{int} \bar{O}=O$.

\section{Construction of Self-COnformal Sets}

For constructing self-conformal sets and measures we need a more complicated probability space.

Let $\Sigma=\{1, \ldots, N\}^{\mathbb{N}}$ be the set of infinite sequences of integers between 1 and $N$, and let $\mathfrak{S}$ be the usual product $\sigma$-algebra on $\Sigma$. Further, let $\Sigma_{n}=\{1, \ldots, N\}^{n}$ be the set of sequences of length $n$, and let $\Sigma_{*}=\bigcup_{n=0}^{\infty} \Sigma_{n}$ be the set of all finite sequences (including the empty sequence $\emptyset$ ). Now define the probability space

$$
\Omega=\Omega_{0}{ }^{\Sigma_{*}},
$$

and let $\mathfrak{F}$ be the corresponding product $\sigma$-algebra. Finally, let $\mathbb{P}$ be the product measure on $\Omega$ with $\mathbb{P}_{0}$ on each component. The elements of this probability space assign to each finite sequence $\tau \in \Sigma_{*}$ a random variable

$$
\omega(\tau)=\left(S_{1}^{\tau}(\omega), \ldots, S_{N}^{\tau}(\omega)\right) .
$$

Write $\omega(\emptyset)=\left(S_{1}(\omega), \ldots, S_{N}(\omega)\right)$. The family of these random variables is a family of independent random variables with identical distribution $\mathbb{P}_{0}$. We need this complicated probability space because of the independence property in the definition of self-conformal sets.

Define operators $T^{\eta}$ on $\Omega$ by

$$
\left(T^{\eta} \omega\right)(\tau)=\omega(\eta \tau)
$$

for $\eta \in \Sigma_{*}$ and $\omega \in \Omega$. By definition, $S_{i}^{\tau}(\omega)=S_{i}\left(T^{\tau} \omega\right)$. It is easy to see that $\mathbb{P}$ is invariant under all these operators. If $\eta, \tau \in \Sigma_{*}$, then $T^{\eta} \circ T^{\tau}=T^{\tau \eta}$.

We say that $\mathbb{P}$ is an iterated conformal random function system if $\mathbb{P}_{0}$ is a conformal random function system.

By definition,

$$
\Omega \cong \Omega_{0} \times \Omega^{N}
$$

with the mapping

$$
\omega \mapsto\left(\omega(\emptyset) ; T^{1} \omega, \ldots, T^{N} \omega\right),
$$

and the image of $\mathbb{P}$ under this mapping is $\mathbb{P}_{0} \times \mathbb{P}^{N}$.

Define $\bar{S}_{\emptyset}^{\tau}=i d_{U}$ and

$$
\bar{S}_{\eta i}^{\tau}=\bar{S}_{\eta}^{\tau} \circ S_{i}^{\tau \eta}
$$

for $\tau, \eta \in \Sigma_{*}$ and $i=1, \ldots, N$. With the notation $\bar{S}_{\eta}^{\emptyset}=\bar{S}_{\eta}$, then $\bar{S}_{\eta}^{\tau}(\omega)=\bar{S}_{\eta}\left(T^{\tau} \omega\right)$ and $\bar{S}_{\tau \eta}=\bar{S}_{\tau} \circ \bar{S}_{\eta}^{\tau}$.

Now we are able to construct the self-conformal random set $\Xi$. 
Define a mapping $\pi: \Omega \times \Sigma \rightarrow U$ by

$$
\pi_{\omega}(\sigma)=\lim _{n \rightarrow \infty} \bar{S}_{\sigma \mid n}(\omega)\left(x_{0}\right)
$$

where $x_{0} \in U$ is an arbitrary point and $\sigma \mid n=\sigma_{1} \sigma_{2} \ldots \sigma_{n}$ is the finite sequence consisting of the first $n$ entries of $\sigma$. This limit exists and does not depend on the choice of $x_{0}$ for $\mathbb{P}$-almost all $\omega \in \Omega$. Moreover, $\pi_{\omega}: \Sigma \rightarrow U$ is continuous for $\mathbb{P}$-almost all $\omega$. Set $\Xi(\omega)=\pi_{\omega}(\Sigma)$.

Lemma 1. $\Xi$ is a self-conformal random set.

Proof. By definition,

$$
\pi_{\omega}(i \sigma)=\lim _{n \rightarrow \infty} \bar{S}_{i \sigma \mid n}(\omega)\left(x_{0}\right)=S_{i}(\omega)\left(\lim _{n \rightarrow \infty} \bar{S}_{\sigma \mid n}\left(T^{i} \omega\right)\left(x_{0}\right)\right)=S_{i}(\omega)\left(\pi_{T^{i} \omega}(\sigma)\right)
$$

for all $i=1, \ldots, N$, all $\sigma \in \Sigma$, and $\mathbb{P}$-almost all $\omega \in \Omega$. Hence,

$$
\Xi(\omega)=\pi_{\omega}(\Sigma)=\bigcup_{i=1}^{N} \pi_{\omega}(i \Sigma)=\bigcup_{i=1}^{N} S_{i}(\omega)\left(\pi_{T^{i} \omega}(\Sigma)\right)=\bigcup_{i=1}^{N} S_{i}(\omega)\left(\Xi\left(T^{i} \omega\right)\right)
$$

for $\mathbb{P}$-almost all $\omega \in \Omega$. By definition of $\Omega$, the random sets $\Xi^{i}(\omega)=\Xi\left(T^{i} \omega\right)$ are independent copies of $\Xi$ and are independent of $\left(S_{1}, \ldots, S_{N}\right)$.

Another way for constructing $\Xi$ is the following. Let $K \subseteq U$ be a compact set with $S_{i}(K) \subseteq K$ for all $i=1, \ldots, N$ with probability one. (If the open set condition (OSC) or the strong open set condition (SOSC) is satisfied one can choose $K=\bar{O}$.) Define

$$
K_{\tau}(\omega)=\bar{S}_{\tau}(\omega)(K)
$$

for $\tau \in \Sigma_{*}$ and $\omega \in \Omega$. Then

$$
\Xi(\omega)=\bigcap_{n=0}^{\infty} \bigcup_{\tau \in \Sigma_{n}} K_{\tau}(\omega)
$$

for $\mathbb{P}$-almost all $\omega \in \Omega$.

\section{The equivalence of the open Set Conditions}

Before proving the main theorem we need some lemmas. Let the open set condition (OSC) be satisfied with the open set $O$ and let $K=\bar{O}$ be compact.

For a conformal mapping $S$ write $\left\|S^{\prime}\right\|=\sup \left\{\left|S^{\prime}(x)\right|: x \in U\right\}$. The Hölder continuity of the derivatives of the mappings $S_{1}, \ldots, S_{N}$ carries over to the mappings $S_{\tau}$ for $\tau \in \Sigma_{*}$.

Lemma 2. Let $\mathbb{P}_{0}$ be an iterated conformal function system. Then there is an open set $U^{\prime} \subseteq U$ with the following properties.

(i) There is a constant $c_{1} \geq 0$ such that

$$
\left|\bar{S}_{\tau}^{\prime}(\omega)(x)\right| \leq\left|\bar{S}_{\tau}^{\prime}(\omega)(y)\right| e^{c_{1} d(x, y)^{\gamma}}
$$

holds for all $x, y \in U^{\prime}$, all $\tau \in \Sigma_{*}$, and $\mathbb{P}$-almost all $\omega \in \Omega$.

(ii) There is a constant $c_{2} \geq 1$ such that

$$
c_{2}^{-1}\left\|\bar{S}_{\tau}^{\prime}(\omega)\right\| d(x, y) \leq d\left(\bar{S}_{\tau}(\omega)(x), \bar{S}_{\tau}(\omega)(y)\right) \leq c_{2}\left\|\bar{S}_{\tau}^{\prime}(\omega)\right\| d(x, y)
$$

holds for all $x, y \in U^{\prime}$, all $\tau \in \Sigma_{*}$, and all $\omega \in \Omega$. 
Proof. We omit the proof here. In $[\mathrm{P} 2]$ the non-random case is considered. The proof there works also in our random setting. Part (i) is a by-product of the proof of [P2, 2.1] and part (ii) is the same as [P2, 2.2].

In the sequel let $U=U^{\prime}$ from the lemma above. A useful tool for the investigation of the random fractal is the notion of a section. We call a random subset $\Gamma \subseteq \Sigma_{*}$ a section if for each $\sigma \in \Sigma$ and each $\omega \in \Omega$ there is a unique $n \in \mathbb{N}$ with $\sigma \mid n \in \Gamma(\omega)$.

A simple example is the random set

$$
\Gamma_{r}=\left\{\tau \in \Sigma_{*}:\left\|\bar{S}_{\tau}^{\prime}\right\| \leq r<\left\|\bar{S}_{\tau|| \tau \mid-1}^{\prime}\right\|\right\}
$$

for $r \in(0,1)$.

It is easy to see that if $\Gamma$ is a section and the open set condition is satisfied, then $\left\{\bar{S}_{\tau}(\omega)(O): \tau \in \Gamma(\omega)\right\}$ is a family of mutually disjoint sets for almost all $\omega \in \Omega$.

Define for $a \in[0,1], b \geq 1$ and $\eta \in \Sigma_{*}$ the random set

$$
\begin{aligned}
& I_{a, b}(\eta)(\omega) \\
& \quad=\left\{\tau \in \Sigma_{*}: \frac{1}{b} \leq \frac{\left\|\bar{S}_{\eta}^{\prime}(\omega)\right\|}{\left\|\bar{S}_{\tau}^{\prime}(\omega)\right\|} \leq b, \operatorname{dist}\left(K_{\tau}(\omega), K_{\eta}(\omega)\right) \leq a\left\|\bar{S}_{\eta}^{\prime}(\omega)\right\|\right\} .
\end{aligned}
$$

Remark that, in general, it is not a section.

Lemma 3. If the open set condition (OSC) is satisfied, then for all $b_{0} \geq 1$ there is a constant $c_{3}=c_{3}\left(b_{0}\right)$ such that

$$
\# I_{a, b}(\eta)(\omega) \leq c_{3}
$$

for all $\eta \in \Sigma_{*}$ and all $a \in[0,1]$ and $b \in\left[b_{0}, 2 b_{0}\right]$ with probability one.

Proof. It is easy to see that for fixed $r>0$

$$
\left\{\tau \in \Sigma_{*}: r_{\max } r \leq\left\|\bar{S}_{\tau}(\omega)\right\|<r\right\} \subseteq \Gamma_{r}(\omega)
$$

holds, where $\Gamma_{r}$ is a section and, in particular, the corresponding sets $\bar{S}_{\tau}(O)$ are disjoint.

Fix $a \in[0,1]$ and $b \in\left[b_{0}, 2 b_{0}\right]$. Let $m$ be the smallest integer such that $r_{\max }^{m}<$ $1 /\left(2 b_{0}^{2}\right)$. Then

with

$$
I_{a, b}(\eta)(\omega) \subseteq \bigcup_{k=0}^{m-1} J_{k}(\eta)(\omega)
$$

$$
\begin{array}{r}
J_{k}(\eta)(\omega)=\left\{\tau \in \Sigma_{*}: \frac{1}{b_{0} r_{\max }{ }^{k}} \leq \frac{\left\|\bar{S}_{\eta}^{\prime}(\omega)\right\|}{\left\|\bar{S}_{\tau}^{\prime}(\omega)\right\|} \leq \frac{1}{b_{0} r_{\max } k+1},\right. \\
\left.\operatorname{dist}\left(K_{\tau}(\omega), K_{\eta}(\omega)\right) \leq a\left\|\bar{S}_{\eta}^{\prime}(\omega)\right\|\right\}
\end{array}
$$

for $k=0, \ldots, m-1$.

Hence, $J_{k}(\eta)(\omega) \subseteq \Gamma_{b_{0} r_{\max }{ }^{k}\left\|\bar{S}_{\eta}^{\prime}(\omega)\right\|}$ with probability one. By the open set condition, all sets $\bar{S}_{\tau}(\omega)(O)$ with $\tau \in J_{k}(\eta)(\omega)$ are disjoint. Since int $K \neq \emptyset$ and $K$ is bounded, there is a point $x_{0} \in K$ and there are numbers $r_{1}, r_{2}>0$ such that

$$
B\left(x_{0}, r_{1}\right) \subseteq \operatorname{int} K \subseteq K \subseteq B\left(x_{0}, r_{2}\right) .
$$

By Lemma 2(ii) we get

$$
B\left(\bar{S}_{\tau}(\omega)\left(x_{0}\right), c_{2}^{-1}\left\|\bar{S}_{\tau}^{\prime}(\omega)\right\| r_{1}\right) \subseteq \bar{S}_{\tau}(\omega)(\operatorname{int} K)
$$


and

$$
\bar{S}_{\tau}(\omega)(K) \subseteq B\left(\bar{S}_{\tau}(\omega)\left(x_{0}\right), c_{2}\left\|\bar{S}_{\tau}^{\prime}(\omega)\right\| r_{2}\right)
$$

for all $\tau \in \Sigma_{*}$. Hence, the sets $\bar{S}_{\tau}(\omega)($ int $K)$ have a Lebesgue measure of at least

$$
c_{2}^{-d}\left\|\bar{S}_{\tau}^{\prime}(\omega)\right\|^{d} r_{1}^{d} \mathcal{L}^{d} B(0,1) \geq c_{2}^{-d} b_{0}^{d} r_{\max }{ }^{m d} r_{1}^{d} \mathcal{L}^{d} B(0,1)\left\|\bar{S}_{\eta}^{\prime}(\omega)\right\|^{d}
$$

for $\tau \in J_{k}(\eta)(\omega)$. On the other hand, all these sets are disjoint and contained in a ball of radius

$$
c_{2}\left\|\bar{S}_{\eta}^{\prime}(\omega)\right\| r_{2}+a\left\|\bar{S}_{\eta}^{\prime}(\omega)\right\|+2 c_{2}\left\|\bar{S}_{\tau}^{\prime}(\omega)\right\| r_{2} \leq\left(c_{2} r_{2}+1+2 c_{2} b_{0} r_{2}\right)\left\|\bar{S}_{\eta}^{\prime}(\omega)\right\|
$$

and center $\bar{S}_{\eta}(\omega)\left(x_{0}\right)$. Therefore, by volume estimating,

$$
\# J_{k}(\eta)(\omega) \leq \frac{\left(c_{2} r_{2}+1+2 c_{2} b_{0} r_{2}\right)^{d}}{\left(c_{2}^{-1} b_{0} r_{\max }{ }^{m} r_{1}\right)^{d}}
$$

for all $k$ and hence

$$
\# I_{a, b}(\eta)(\omega) \leq m \frac{\left(c_{2} r_{2}+1+2 c_{2} b_{0} r_{2}\right)^{d}}{\left(c_{2}^{-1} b_{0} r_{\max }{ }^{m} r_{1}\right)^{d}},
$$

concluding the proof.

Lemma 4. Let $b_{0} \geq 1$ and $\varepsilon>0$. If the open set condition is satisfied, there exists a constant $\delta>0$ with the following property. Let $a \in[0,1]$ and $b \in\left[b_{0}, 2 b_{0}\right]$. For $\mathbb{P}$-almost all $\omega \in \Omega$, if $\tau, \eta \in \Sigma_{*}$ are such that $\left\|\left(\bar{S}_{\eta}^{\tau}\right)^{\prime}(\omega)\right\| \leq \delta$, then $\tau \xi \in$ $I_{a(1+\varepsilon), b(1+\varepsilon)}(\tau \eta)(\omega)$ for all $\xi \in I_{a, b}(\eta)\left(T^{\tau} \omega\right)$.

Proof. Choose $\delta>0$ such that $B(x, \delta) \subseteq U$ for each $x \in K$ and

$$
c_{1} \delta^{\gamma}\left(\left(a+c_{2} \operatorname{diam} U\right)^{\gamma}+\left(2 b_{0} c_{2} \operatorname{diam} U\right)^{\gamma}\right) \leq \log (1+\varepsilon)
$$

hold.

Let the assumptions of the lemma be satisfied, and let $\tau, \eta \in \Sigma_{*}$ with $\left\|\left(\bar{S}_{\eta}^{\tau}\right)^{\prime}(\omega)\right\|$ $\leq \delta$ and $\xi \in I_{a, b}(\eta)\left(T^{\tau} \omega\right)$. Since $\operatorname{dist}\left(K_{\eta}^{\tau}(\omega), K_{\xi}^{\tau}(\omega)\right) \leq a\left\|\left(\bar{S}_{\eta}^{\tau}\right)^{\prime}(\omega)\right\| \leq a \delta$ there exists a point $z_{0} \in K_{\xi}^{\tau}(\omega)$ with

$$
\operatorname{dist}\left(z_{0}, \bar{S}_{\eta}^{\tau}(\omega)(U)\right) \leq \operatorname{dist}\left(z_{0}, K_{\eta}^{\tau}(\omega)\right) \leq a\left\|\left(\bar{S}_{\eta}^{\tau}\right)^{\prime}(\omega)\right\| \leq a \delta .
$$

Hence

$$
d\left(z_{0}, \bar{S}_{\eta}^{\tau}(\omega)\left(x_{0}\right)\right) \leq a \delta+c_{2} \operatorname{diam} U\left\|\left(\bar{S}_{\eta}^{\tau}\right)^{\prime}(\omega)\right\| \leq\left(a+c_{2} \operatorname{diam} U\right) \delta
$$

for all $x_{0} \in U$ by Lemma 2(ii) implying

$$
\begin{aligned}
\left|\bar{S}_{\tau \eta}^{\prime}(\omega)\left(x_{0}\right)\right| & =\left|\bar{S}_{\tau}^{\prime}(\omega)\left(\bar{S}_{\eta}^{\tau}(\omega)\left(x_{0}\right)\right)\right|\left|\left(\bar{S}_{\eta}^{\tau}\right)^{\prime}(\omega)\left(x_{0}\right)\right| \\
& \leq\left|\bar{S}_{\tau}^{\prime}(\omega)\left(z_{0}\right)\right|\left\|\left(\bar{S}_{\eta}^{\tau}\right)^{\prime}(\omega)\right\| e^{c_{1}\left(a+c_{2} \operatorname{diam} U\right)^{\gamma} \delta^{\gamma}}
\end{aligned}
$$

for all $x_{0} \in U$ by Lemma $2(\mathrm{i})$ and, therefore,

$$
\left\|\bar{S}_{\tau \eta}^{\prime}(\omega)\right\| \leq\left|\bar{S}_{\tau}^{\prime}(\omega)\left(z_{0}\right)\right|\left\|\left(\bar{S}_{\eta}^{\tau}\right)^{\prime}(\omega)\right\| e^{c_{1}\left(a+c_{2} \operatorname{diam} U\right)^{\gamma} \delta^{\gamma}} .
$$

On the other hand,

$$
\begin{aligned}
\left\|\bar{S}_{\tau \eta}^{\prime}(\omega)\right\| & \geq\left|\bar{S}_{\tau \eta}^{\prime}(\omega)\left(x_{0}\right)\right| \\
& =\left|\bar{S}_{\tau}^{\prime}(\omega)\left(\bar{S}_{\eta}^{\tau}(\omega)\left(x_{0}\right)\right)\right|\left|\left(\bar{S}_{\eta}^{\tau}\right)^{\prime}(\omega)\left(x_{0}\right)\right| \\
& \geq\left|\bar{S}_{\tau}^{\prime}(\omega)\left(z_{0}\right)\right|\left|\left(\bar{S}_{\eta}^{\tau}\right)^{\prime}(\omega)\left(x_{0}\right)\right| e^{-c_{1}\left(a+c_{2} \operatorname{diam} U\right)^{\gamma} \delta^{\gamma}}
\end{aligned}
$$


for all $x_{0} \in U$ implying

$$
\left\|\bar{S}_{\tau \eta}^{\prime}(\omega)\right\| \geq\left|\bar{S}_{\tau}^{\prime}(\omega)\left(z_{0}\right)\right|\left\|\left(\bar{S}_{\eta}^{\tau}\right)^{\prime}(\omega)\right\| e^{-c_{1}\left(a+c_{2} \operatorname{diam} U\right)^{\gamma} \delta^{\gamma}} .
$$

Since

$$
\begin{aligned}
\operatorname{diam}\left(S_{\xi}^{\tau}(\omega)(U)\right) & \leq c_{2} \operatorname{diam} U\left\|\left(\bar{S}_{\xi}^{\tau}\right)^{\prime}(\omega)\right\| \\
& \leq c_{2} \operatorname{diam} U \cdot b\left\|\left(\bar{S}_{\eta}^{\tau}\right)^{\prime}(\omega)\right\| \\
& \leq c_{2} \operatorname{diam} U \cdot 2 b_{0} \delta
\end{aligned}
$$

and $z_{0} \in K_{\xi}^{\tau}(\omega) \subseteq S_{\xi}^{\tau}(\omega)(U)$, the inequalities

$$
\begin{aligned}
& \left|\bar{S}_{\tau}^{\prime}(\omega)\left(z_{0}\right)\right|\left\|\left(\bar{S}_{\xi}^{\tau}\right)^{\prime}(\omega)\right\| e^{-c_{1}\left(2 b_{0} c_{2} \operatorname{diam} U\right)^{\gamma} \delta^{\gamma}} \\
& \quad \leq\left\|\bar{S}_{\tau \xi}^{\prime}(\omega)\right\| \\
& \quad \leq\left|\bar{S}_{\tau}^{\prime}(\omega)\left(z_{0}\right)\right|\left\|\left(\bar{S}_{\xi}^{\tau}\right)^{\prime}(\omega)\right\| e^{c_{1}\left(2 b_{0} c_{2} \operatorname{diam} U\right)^{\gamma} \delta^{\gamma}}
\end{aligned}
$$

hold. By the assumption that $\xi \in I_{a, b}(\eta)\left(T^{\tau} \omega\right)$, the inequalities

$$
b^{-1} \leq \frac{\left\|\left(\bar{S}_{\eta}^{\tau}\right)^{\prime}(\omega)\right\|}{\left\|\left(\bar{S}_{\xi}^{\tau}\right)^{\prime}(\omega)\right\|} \leq b
$$

are satisfied implying

$$
\begin{aligned}
\frac{\left\|\bar{S}_{\tau \eta}^{\prime}(\omega)\right\|}{\left\|\bar{S}_{\tau \xi}^{\prime}(\omega)\right\|} & \leq \frac{\left|\bar{S}_{\tau}^{\prime}(\omega)\left(z_{0}\right)\right|\left\|\left(\bar{S}_{\eta}^{\tau}\right)^{\prime}(\omega)\right\| e^{c_{1}\left(a+c_{2} \operatorname{diam} U\right)^{\gamma} \delta^{\gamma}}}{\left|\bar{S}_{\tau}^{\prime}(\omega)\left(z_{0}\right)\right|\left\|\left(\bar{S}_{\xi}^{\tau}\right)^{\prime}(\omega)\right\| e^{-c_{1}\left(2 b_{0} c_{2} \operatorname{diam} U\right)^{\gamma} \delta^{\gamma}}} \\
& \leq b(1+\varepsilon)
\end{aligned}
$$

and

$$
\frac{\left\|\bar{S}_{\tau \eta}^{\prime}(\omega)\right\|}{\left\|\bar{S}_{\tau \xi}^{\prime}(\omega)\right\|} \geq \frac{1}{b(1+\varepsilon)}
$$

by definition of $\delta$, which is the first property to be checked for the definition of $I_{a(1+\varepsilon), b(1+\varepsilon)}(\tau \eta)(\omega)$.

Since $\xi \in I_{a, b}(\eta)\left(T^{\tau} \omega\right)$ there are $x, y \in K$ with

$$
d\left(\bar{S}_{\eta}^{\tau}(\omega)(x), \bar{S}_{\xi}^{\tau}(\omega)(y)\right) \leq a\left\|\left(\bar{S}_{\eta}^{\tau}\right)^{\prime}(\omega)\right\| \leq \delta .
$$

Therefore, the line joining $\bar{S}_{\eta}^{\tau}(\omega)(x)$ and $\bar{S}_{\xi}^{\tau}(\omega)(y)$ is contained in U. So there is a point $z_{1} \in U$ with $\operatorname{dist}\left(z_{1}, K_{\eta}^{\tau}(\omega)\right) \leq a\left\|\left(\bar{S}_{\eta}^{\tau}\right)^{\prime}(\omega)\right\|$ such that

$$
\begin{aligned}
d\left(\bar{S}_{\tau \eta}(\omega)(x), \bar{S}_{\tau \xi}(\omega)(y)\right) & =\left|\bar{S}_{\tau}^{\prime}\left(z_{1}\right)(\omega)\right| d\left(\bar{S}_{\eta}^{\tau}(\omega)(x), \bar{S}_{\xi}^{\tau}(\omega)(y)\right) \\
& \leq a\left|\bar{S}_{\tau}^{\prime}(\omega)\left(z_{1}\right)\right|\left\|\left(\bar{S}_{\eta}^{\tau}\right)^{\prime}(\omega)\right\|
\end{aligned}
$$

Let $x_{0} \in U$. Then

$$
\begin{aligned}
d\left(z_{1}, \bar{S}_{\eta}^{\tau}(\omega)\left(x_{0}\right)\right) & \leq a\left\|\left(\bar{S}_{\eta}^{\tau}\right)^{\prime}(\omega)\right\|+c_{2} \operatorname{diam} U\left\|\left(\bar{S}_{\eta}^{\tau}\right)^{\prime}(\omega)\right\| \\
& \leq\left(a+c_{2} \operatorname{diam} U\right) \delta .
\end{aligned}
$$

This implies

$$
\begin{aligned}
& \left|\bar{S}_{\tau}^{\prime}(\omega)\left(z_{1}\right)\right|\left|\left(\bar{S}_{\eta}^{\tau}\right)^{\prime}(\omega)\left(x_{0}\right)\right| \\
& \quad \leq\left|\bar{S}_{\tau}^{\prime}(\omega)\left(\bar{S}_{\eta}^{\tau}(\omega)\left(x_{0}\right)\right)\right|\left|\left(\bar{S}_{\eta}^{\tau}\right)^{\prime}(\omega)\left(x_{0}\right)\right| e^{c_{1}\left(a+c_{2} \operatorname{diam} U\right)^{\gamma} \delta^{\gamma}} \\
& \quad \leq\left\|\bar{S}_{\tau \eta}^{\prime}(\omega)\right\| e^{c_{1}\left(a+c_{2} \operatorname{diam} U\right)^{\gamma} \delta^{\gamma}}
\end{aligned}
$$


for all $x_{0} \in U$ and, hence,

$$
\left|\bar{S}_{\tau}^{\prime}(\omega)\left(z_{1}\right)\right|\left\|\left(\bar{S}_{\eta}^{\tau}\right)^{\prime}(\omega)\right\| \leq\left\|\bar{S}_{\tau \eta}^{\prime}(\omega)\right\| e^{c_{1}\left(a+c_{2} \operatorname{diam} U\right)^{\gamma} \delta^{\gamma}}
$$

and

$$
\begin{aligned}
\operatorname{dist}\left(K_{\tau \eta}(\omega), K_{\tau \xi}(\omega)\right) & \leq d\left(\bar{S}_{\tau \eta}(\omega)(x), \bar{S}_{\tau \xi}(\omega)(y)\right) \\
& \leq a\left\|\bar{S}_{\tau \eta}^{\prime}(\omega)\right\| e^{c_{1}\left(a+c_{2} \operatorname{diam} U\right)^{\gamma} \delta^{\gamma}} \\
& \leq a(1+\varepsilon)\left\|\bar{S}_{\tau \eta}^{\prime}(\omega)\right\|
\end{aligned}
$$

which is the second property in the definition of $I_{a(1+\varepsilon), b(1+\varepsilon)}(\tau \eta)(\omega)$, completing the proof.

Now, we prove a random version of the strong open set condition, as can be found for the self-similar case in [P1] Theorem 3].

Lemma 5. If the open set condition is satisfied, there exists a random open set $G$ such that the following hold.

(i) $S_{i}\left(G^{i}\right) \subseteq G$ for all $i=1, \ldots, N$ with probability one (where $G^{i}(\omega)=$ $\left.G\left(T^{i} \omega\right)\right)$.

(ii) $S_{i}\left(G^{i}\right) \cap S_{j}\left(G^{j}\right)=\emptyset$ for all $i \neq j$ with probability one.

(iii) $\Xi \cap G \neq \emptyset$ with probability one.

Proof. 1. Fix

$$
b_{0}=\max \left\{\sqrt{C r_{\min }^{-1}}, r_{\min }^{-1}\right\} .
$$

Then $\left\|\bar{S}_{\xi}^{\prime}\right\| \leq b_{0}^{2}\left\|\bar{S}_{\xi j}^{\prime}\right\|$ and $b_{0}\left\|S_{j}^{\prime}\right\| \geq 1$ hold for all $j=1, \ldots, N$ with probability one. Moreover, if $r \leq 1$ and $\tau=\tau_{1} \ldots \tau_{n} \in \Sigma_{*}$ with $\left\|\bar{S}_{\tau}^{\prime}\right\| \leq r$, then there exists an integer $k \leq n$ such that

holds.

$$
r b_{0}^{-1} \leq\left\|\bar{S}_{\tau \mid k}^{\prime}\right\| \leq r b_{0}
$$

Let $M_{a}(\eta)=\# I_{a,(1+a) b_{0}}(\eta)$. By Lemma 3 there is a constant $c_{3}$ such that $M_{a}(\eta) \leq c_{3}$ for all $\eta \in \Sigma_{*}$ and all $a \in[0,1]$ with probability one. The function $a \mapsto M_{a}(\eta)(\omega)$ is non-decreasing for all $\omega \in \Omega$ and all $\eta \in \Sigma_{*}$. Define

$$
L_{a}(r)=\operatorname{ess} \sup \sup \left\{M_{a}(\eta): \eta \in \Sigma_{*},\left\|\bar{S}_{\eta}^{\prime}\right\| \leq r\right\}
$$

for $r \leq 1$. By Lemma 3 this value is well defined and finite. Let $\varepsilon=1 /\left(2 c_{3}\right)$ and let $\delta$ be such that Lemma 4 holds with these numbers. Without loss of generality we may assume that $\delta \leq 1$. The function $a \mapsto L_{a}(\delta)$ on $[0,1]$ is non-decreasing, integer valued, and bounded by $c_{3}$. Hence, there exists an interval $\left[a_{1}, a_{2}\right] \subseteq[0,1]$ with $a_{2}-a_{1} \geq c_{3}^{-1}$ such that $L_{a_{1}}(\delta)=L_{a_{2}}(\delta)$. Since the random value $M_{a_{1}}(\eta)$ is integer valued, there is an $\eta \in \Sigma_{*}$ with

$$
\mathbb{P}\left(\left\|\bar{S}_{\eta}^{\prime}\right\| \leq \delta, M_{a_{1}}(\eta)=L_{a_{1}}(\delta)\right)>0 .
$$

Define

$$
\Upsilon=\left\{(\omega, \eta) \in \Omega \times \Sigma_{*}:\left\|\bar{S}_{\eta}^{\prime}(\omega)\right\| \leq \delta, M_{a_{1}}(\eta)(\omega)=L_{a_{1}}(\delta)\right\}
$$

and let $\Omega^{\prime}$ be the projection onto the first component. Then $\mathbb{P}\left(\Omega^{\prime}\right)>0$. If $(\omega, \eta) \in$ $\Upsilon$, then

$$
M_{a_{1}}(\eta)(\omega)=M_{a_{2}}(\eta)(\omega)=L_{a_{1}}(\delta)=L_{a_{2}}(\delta)
$$

holds. 
2. By definition,

$$
a_{2} \geq a_{1}+c_{3}^{-1} \geq a_{1}\left(1+1 /\left(2 c_{3}\right)\right)=a_{1}(1+\varepsilon)
$$

and

$$
\begin{aligned}
1+a_{2} & \geq 1+a_{1}+\left(1+a_{1}\right) /\left(2 c_{3}\right) \\
& =\left(1+a_{1}\right)\left(1+1 /\left(2 c_{3}\right)\right)=\left(1+a_{1}\right)(1+\varepsilon)
\end{aligned}
$$

are satisfied. Hence, if $\xi \in I_{a_{1},\left(1+a_{1}\right) b_{0}}(\eta)\left(T^{\tau} \omega\right)$, then

$$
\tau \xi \in I_{a_{1}(1+\varepsilon),\left(1+a_{1}\right) b_{0}(1+\varepsilon)}(\tau \eta)(\omega) \subseteq I_{a_{2},\left(1+a_{2}\right) b_{0}}(\tau \eta)(\omega)
$$

by Lemma 4. Consequently, $M_{a_{2}}(\tau \eta)(\omega) \geq M_{a_{1}}(\eta)\left(T^{\tau} \omega\right)$. If $\left(\eta, T^{\tau} \omega\right) \in \Upsilon$, then

$$
M_{a_{2}}(\tau \eta)(\omega) \leq L_{a_{2}}(\delta)=L_{a_{1}}(\delta)=M_{a_{1}}(\eta)\left(T^{\tau} \omega\right),
$$

which implies

$$
M_{a_{2}}(\eta)\left(T^{\tau} \omega\right)=M_{a_{1}}(\eta)\left(T^{\tau} \omega\right)=M_{a_{2}}(\tau \eta)(\omega) .
$$

Therefore

$$
\begin{aligned}
I_{a_{2},\left(1+a_{2}\right) b_{0}}(\tau \eta)(\omega) & =\left\{\tau \xi: \xi \in I_{a_{1},\left(1+a_{1}\right) b_{0}}(\eta)\left(T^{\tau} \omega\right)\right\} \\
& =\tau I_{a_{1},\left(1+a_{1}\right) b_{0}}(\eta)\left(T^{\tau} \omega\right)
\end{aligned}
$$

and

3. Recall

$$
M_{a_{2}}(\tau \eta)(\omega)=M_{a_{2}}(\eta)\left(T^{\tau} \omega\right)=L_{a_{1}}(\delta)=M_{a_{1}}(\eta)(\omega) .
$$

$$
\Omega^{\prime}=\left\{\omega \in \Omega: \exists \eta \in \Sigma_{*} \text { with }\left\|\bar{S}_{\eta}^{\prime}(\omega)\right\| \leq \delta, M_{a_{1}}(\eta)(\omega)=L_{a_{1}}(\delta)\right\}
$$

and define

$$
\Omega^{\prime \prime}=\left\{\omega \in \Omega: \exists \eta \in \Sigma_{*} \text { with }\left\|\bar{S}_{\eta}^{\prime}(\omega)\right\| \leq \delta, M_{a_{2}}(\eta)(\omega)=L_{a_{1}}(\delta)\right\} .
$$

By step $2,\left(T^{\tau}\right)^{-1} \Omega^{\prime} \subseteq \Omega^{\prime \prime}$ for all $\tau \in \Sigma_{*}$. For integer $n$ the family

$$
\left\{\left(T^{\tau}\right)^{-1} \Omega^{\prime}: \tau \in \Sigma_{n}\right\}
$$

is independent. Let $p_{1}=\mathbb{P}\left(\Omega^{\prime}\right)$ and $p_{2}=\mathbb{P}\left(\Omega^{\prime \prime}\right)$. Then $0<p_{1} \leq p_{2}$. Hence,

$$
\begin{aligned}
p_{2} & =\mathbb{P}\left(\Omega^{\prime \prime}\right) \\
& \geq \mathbb{P}\left(\bigcup_{\tau \in \Sigma_{n}}\left(T^{\tau}\right)^{-1} \Omega^{\prime}\right) \\
& =1-\mathbb{P}\left(\bigcap_{\tau \in \Sigma_{n}}\left(T^{\tau}\right)^{-1}\left(\Omega^{\prime}\right)^{c}\right) \\
& =1-\left(1-p_{1}\right)^{N^{n}}
\end{aligned}
$$

for all $n \geq 1$. This implies $1-p_{2} \leq\left(1-p_{1}\right)^{N^{n}}$ for all $n \geq 1$. But $p_{1}>0$, therefore $p_{2}=1$. Set

$$
\bar{\Omega}=\left\{\omega \in \Omega: T^{\tau} \omega \in \Omega^{\prime \prime} \text { for all } \tau \in \Sigma_{*}\right\} .
$$

Since $\Sigma_{*}$ is countable, we have $\mathbb{P}(\bar{\Omega})=1$. By definition, $\bar{\Omega}$ is invariant under all $T^{\tau}, \tau \in \Sigma_{*}$, and for each $\omega \in \bar{\Omega}$ there is an $\eta \in \Sigma_{*}$ such that $\left\|\bar{S}_{\eta}^{\prime}(\omega)\right\| \leq \delta$ and $M_{a_{2}}(\eta)(\omega)=L_{a_{1}}(\delta)$. We may assume that the mapping $\omega \mapsto \eta(\omega)$ is measurable.

4. Set $\varepsilon^{\prime}=\min \left(a_{2} /\left(2 c_{2}\right), \operatorname{dist}\left(K, U^{c}\right)\right)$ and define

$$
G(\omega)=\bigcup_{\tau \in \Sigma_{*}} \bar{S}_{\tau}(\omega) \circ \bar{S}_{\eta\left(T^{\tau} \omega\right)}\left(T^{\tau} \omega\right)\left(B\left(K, \varepsilon^{\prime}\right)\right)
$$


for $\omega \in \bar{\Omega}$, where $B\left(K, \varepsilon^{\prime}\right)=\left\{x \in U: \operatorname{dist}(x, K)<\varepsilon^{\prime}\right\}\left(\right.$ remark: $\left.\bar{S}_{\eta}^{\tau}(\omega)=\bar{S}_{\eta}\left(T^{\tau} \omega\right)\right)$. It remains to show that $G$ satisfies the assertions.

(i) If $\omega \in \bar{\Omega}$, then

$$
\begin{aligned}
S_{i}(\omega)\left(G^{i}(\omega)\right) & =S_{i}(\omega)\left(\bigcup_{\tau \in \Sigma_{*}} \bar{S}_{\tau}\left(T^{i} \omega\right) \circ \bar{S}_{\eta\left(T^{i \tau} \omega\right)}\left(T^{i \tau} \omega\right)\left(B\left(K, \varepsilon^{\prime}\right)\right)\right) \\
& =\bigcup_{\tau \in \Sigma_{*}} \bar{S}_{i \tau}(\omega) \circ \bar{S}_{\eta^{i \tau}(\omega)}^{i \tau}(\omega)\left(B\left(K, \varepsilon^{\prime}\right)\right) \subseteq G(\omega)
\end{aligned}
$$

for all $i=1, \ldots, N$ since $T^{\tau} \circ T^{i}=T^{i \tau}$.

(ii) Assume there is an $\omega \in \bar{\Omega}$ and a pair $i \neq j$ with $S_{i}\left(G^{i}(\omega)\right) \cap S_{j}\left(G^{j}(\omega)\right) \neq \emptyset$. By definition of $G$ there are $\tau_{1}, \tau_{2} \in \Sigma_{*}$ and $\eta_{1}=\eta\left(T^{i \tau_{1}} \omega\right)$ and $\eta_{2}=\eta\left(T^{j \tau_{2}} \omega\right)$ such that there is a $y \in \bar{S}_{i \tau_{1}}(\omega) \circ \bar{S}_{\eta_{1}}^{i \tau_{1}}(\omega)\left(B\left(K, \varepsilon^{\prime}\right)\right) \cap \bar{S}_{j \tau_{2}}(\omega) \circ \bar{S}_{\eta_{2}}^{j \tau_{2}}(\omega)\left(B\left(K, \varepsilon^{\prime}\right)\right)$. Without loss of generality we assume $\left\|\bar{S}_{j \tau_{2} \eta_{2}}(\omega)\right\| \leq\left\|\bar{S}_{i \tau_{1} \eta_{1}}(\omega)\right\|$. By our choice of $b_{0}$ we can write $\tau_{2} \eta_{2}=\tau^{\prime} \tau^{\prime \prime}$ such that

$$
b_{0}^{-1} \leq \frac{\left\|\bar{S}_{i \tau_{1} \eta_{1}}(\omega)\right\|}{\left\|\bar{S}_{j \tau^{\prime}}(\omega)\right\|} \leq b_{0} .
$$

Hence, $j \tau^{\prime}$ satisfies the first condition of the definition of $I_{a_{2},\left(1+a_{2}\right) b_{0}}\left(i \tau_{1} \eta_{1}\right)(\omega)$. By definition there are $y_{1} \in K_{i \tau_{1} \eta}(\omega)$ with

$$
d\left(y_{1}, y\right) \leq c_{2} \varepsilon^{\prime}\left\|\bar{S}_{i \tau_{1} \eta_{1}}^{\prime}(\omega)\right\|
$$

and $y_{2} \in K_{j \tau^{\prime}}(\omega)$ with

$$
d\left(y_{2}, y\right) \leq c_{2} \varepsilon^{\prime}\left\|\bar{S}_{j \tau_{2} \eta_{2}}^{\prime}(\omega)\right\| \leq c_{2} \varepsilon^{\prime}\left\|\bar{S}_{i \tau_{1} \eta_{1}}^{\prime}(\omega)\right\| .
$$

Hence,

$$
\operatorname{dist}\left(K_{j \tau^{\prime}}(\omega), K_{i \tau_{1} \eta_{1}}(\omega)\right) \leq 2 c_{2} \varepsilon^{\prime}\left\|\bar{S}_{i \tau_{1} \eta_{1}}^{\prime}(\omega)\right\| \leq a_{2}\left\|\bar{S}_{i \tau_{1} \eta_{1}}^{\prime}(\omega)\right\|
$$

which is the second condition in the definition of $I_{a_{2},\left(1+a_{2}\right) b_{0}}\left(i \tau_{1} \eta_{1}\right)(\omega)$. Hence, $j \tau^{\prime} \in I_{a_{2},\left(1+a_{2}\right) b_{0}}\left(i \tau_{1} \eta_{1}\right)(\omega)$, a contradiction to step 3 .

(iii) Clearly, $K_{\eta(\omega)}(\omega) \subseteq G(\omega)$ for all $\omega \in \bar{\Omega}$ and, hence, $\Xi(\omega) \cap G(\omega) \neq \emptyset$.

By means of the lemma above we can prove the existence of a non-random open set satisfying the strong open set condition.

Theorem 6. Let $\mathbb{P}_{0}$ be a conformal random function system satisfying the open set condition. Then the strong open set condition is satisfied.

Proof. Let $\mathbb{P}$ be the corresponding iterated conformal random function system, and let $G$ be the random set from Lemma 5 . Choose a countable basis $\mathcal{B}$ for the topology and define

$$
\mathcal{B}_{1}=\{B \in \mathcal{B}: \mathbb{P}(B \subseteq G)>0\}
$$

Set

$$
O_{1}=\bigcup_{B \in \mathcal{B}_{1}} B
$$

and $O=\operatorname{int} \bar{O}_{1}$. It is clear that $O$ is a non-empty open set and $O=\operatorname{int} \bar{O}$. We have to show that the conditions of the strong open set condition are satisfied. Instead of proving the assertions for $O$ it suffices to show that $O_{1}$ satisfies the conditions of the strong open set condition. By standard arguments it follows that $O$ also satisfies these conditions. Remark that, by definition, $G \subseteq O_{1} \subseteq O$ with probability one. 
Let $B \in \mathcal{B}_{1}$. By independence of $S_{i}$ and $G^{i}$,

$$
\begin{aligned}
\mathbb{P}\left(S_{i}(B) \subseteq O_{1}\right) \mathbb{P}\left(B \subseteq G^{i}\right) & =\mathbb{P}\left(S_{i}(B) \subseteq O_{1}, B \subseteq G^{i}\right) \\
& \geq \mathbb{P}\left(S_{i}(B) \subseteq G, B \subseteq G^{i}\right) \\
& \geq \mathbb{P}\left(S_{i}\left(G^{i}\right) \subseteq G, B \subseteq G^{i}\right) \\
& =\mathbb{P}\left(B \subseteq G^{i}\right),
\end{aligned}
$$

because $\mathbb{P}\left(S_{i}\left(G^{i}\right) \subseteq G\right)=1$. Since $\mathbb{P}\left(B \subseteq G^{i}\right)=\mathbb{P}(B \subseteq G)>0$, this implies $\mathbb{P}\left(S_{i}(B) \subseteq O_{1}\right)=1$ and hence $\mathbb{P}_{0}\left(S_{i}(B) \subseteq O_{1}\right)=1$. Since $\mathcal{B}$ is countable, we get

$$
\begin{aligned}
\mathbb{P}_{0}\left(S_{i}\left(O_{1}\right) \subseteq O_{1}\right) & =\mathbb{P}_{0}\left(\bigcup_{B \in \mathcal{B}_{1}} S_{i}(B) \subseteq O_{1}\right) \\
& =\mathbb{P}_{0}\left(\bigcap_{B \in \mathcal{B}_{1}}\left\{S_{i}(B) \subseteq O_{1}\right\}\right)=1
\end{aligned}
$$

for the open set $O_{1}$.

Let $B, B^{\prime} \in \mathcal{B}_{1}$. By independence and Lemma 5

$$
\begin{aligned}
& \mathbb{P}\left(S_{i}(B) \cap S_{j}\left(B^{\prime}\right)=\emptyset\right) \mathbb{P}\left(B \subseteq G^{i}\right) \mathbb{P}\left(B^{\prime} \subseteq G^{j}\right) \\
& \quad=\mathbb{P}\left(S_{i}(B) \cap S_{j}\left(B^{\prime}\right)=\emptyset, B \subseteq G^{i}, B^{\prime} \subseteq G^{j}\right) \\
& \quad \geq \mathbb{P}\left(S_{i}\left(G^{i}\right) \cap S_{j}\left(G^{j}\right)=\emptyset, B \subseteq G^{i}, B^{\prime} \subseteq G^{j}\right) \\
& \quad=\mathbb{P}\left(B \subseteq G^{i}, B^{\prime} \subseteq G^{j}\right) \\
& \quad=\mathbb{P}\left(B \subseteq G^{i}\right) \mathbb{P}\left(B^{\prime} \subseteq G^{j}\right),
\end{aligned}
$$

which, again, implies $\mathbb{P}_{0}\left(S_{i}(B) \cap S_{j}\left(B^{\prime}\right)=\emptyset\right)=1$ and $\mathbb{P}_{0}\left(S_{i}\left(O_{1}\right) \cap S_{j}\left(O_{1}\right)=\emptyset\right)=1$ by definition of $O_{1}$, proving (ii).

It remains to show that $\Xi \cap O_{1} \neq \emptyset$ with probability one. Let

$$
\Omega^{\prime}=\{\omega \in \Omega: \Xi(\omega) \cap G(\omega) \neq \emptyset\} .
$$

By Lemma $5 \mathbb{P}\left(\Omega^{\prime}\right)=1$. Since $\mathcal{B}$ forms a basis for the topology, for each $\omega \in \Omega^{\prime}$ there is a set $B(\omega) \in \mathcal{B}$ with $\Xi(\omega) \cap B(\omega) \neq \emptyset$ and $B(\omega) \subseteq G(\omega)$. Since $G$ is measurable, we may assume that the mapping $\omega \mapsto B(\omega)$ is measurable. Let $E \in \mathcal{B}$. Then $\mathbb{P}\{\omega \in \Omega: B(\omega)=E\} \leq \mathbb{P}\{\omega \in \Omega: E \subseteq G(\omega)\}$. Hence, $\mathbb{P}\{\omega \in \Omega: B(\omega)=E\}=0$ for all $E \in \mathcal{B} \backslash \mathcal{B}_{1}$. Therefore, $\mathbb{P}\left\{\omega \in \Omega: B(\omega) \notin \mathcal{B}_{1}\right\}=0$ implying $\Xi \cap O_{1} \neq \emptyset$ with probability one.

\section{REFERENCES}

[AP] M. Arbeiter and N. Patzschke, Random self-similar multifractals, Math. Nachr. 181 (1996), 5 - 42. MR 97j:28016

[F] K. J. Falconer, Random fractals, Math. Proc. Cambridge Philos. Soc. 100 (1986), $559-$ 582. MR 88e:28005

[G] S. Graf, Statistically self-similar fractals, Probab. Theory Related Fields 74 (1987), 357 - 392. MR 88c:60038

[LRY] K.-S. Lau, H. Rao, and Y.-L. Ye, Corrigendum to: Iterated function systems and Ruelle Operator, J. Math. Anal. Appl. 262, (2001), 446 - 451. MR 2002g:37010

[MW] R. D. Mauldin and S. C. Williams, Random recursive constructions: asymptotic geometric and topological properties, Trans. Amer. Math. Soc. 295 (1986), 325 - 346. MR 87j:60027

[P1] N. Patzschke, The strong open set condition in the random case, Proc. Amer. Math. Soc. 125 (1997), 2119 - 2125. MR 97i:28005

[P2] N. Patzschke, Self-conformal multifractal measures, Adv. in Appl. Math. 19 (1997), 486 - 513. MR 99c:28020 
[PRSS] Y. Peres, M. Rams, K. Simon, and B. Solomyak, Equivalence of positive Hausdorff measure and the open set condition for self-conformal sets, Proc. Amer. Math. Soc. 129 (2001), 2689 - 2699. MR 2002d:28004

[S] A. Schief, Separation properties for self-similar sets, Proc. Amer. Math. Soc. 122 (1994), 111 - 115. MR 94k:28012

Fakultät für Mathematik und Informatik, Friedrich-Schiller-Universität Jena, D07740 Jena, Germany

E-mail address: patzschke@mathematik.uni-jena.de 